\title{
CONSCIÊNCIA FONOLÓGICA: O DESEMPENHO DE MENINOS E MENINAS BILÍNGUES E MONOLÍNGUES
}

\author{
Phonological awareness: bilingual and monolingual \\ boys and girls performance
}

\author{
Sabrina Schützenhofer Lasch ${ }^{(1)}$, Helena Bolli Mota ${ }^{(2)}$, Carla Aparecida Cielo ${ }^{(3)}$
}

\begin{abstract}
RESUMO
Objetivo: investigar o desempenho de meninas e meninos, monolíngues e bilíngues, em habilidades de consciência fonológica. Métodos: questionário e triagem auditiva para selecionar a amostra, composta por 89 estudantes, faixa etária entre 4:1 a 8:11 anos de idade, 47 meninas e 42 meninos, dos quais 47 sujeitos eram bilíngues (português e alemão) e 42 monolíngues (português). A amostra selecionada foi submetida à avaliação das habilidades em consciência fonológica, com aplicação do Protocolo de Consciência Fonológica (Cielo, 2001) que propõe tarefas de segmentação de frases em palavras; realismo nominal; detecção de rimas; síntese silábica; segmentação silábica; detecção de sílabas; reversão silábica; exclusão fonêmica; detecção de fonemas; síntese fonêmica; segmentação fonêmica e reversão fonêmica. Para uma análise quantitativa dos dados, realizou-se o teste estatístico de Kruskal-Wallis com $\mathrm{p}=0,05$ quando se comparou o desempenho de meninos e meninas monolíngues, bem como o desempenho de meninos e meninas bilíngues por tarefa de CF. Resultados: quando analisados os sujeitos bilíngues, houve significância estatística na detecção de rima com trissílabos $(p=0,0087)$ e na síntese de quatro fonemas $(p=0,0219)$, com vantagem das meninas; já na análise que comparou meninos e meninas monolíngues, os resultados foram mais equilibrados. Conclusões: apesar de praticamente não existirem resultados estatisticamente significantes na comparação entre meninos e meninas bilíngues e monolíngues, observou-se superioridade feminina na maioria das tarefas de consciência fonológica.
\end{abstract}

DESCRITORES: Linguagem; Estudos de Linguagem; Multilinguismo; Conscientização; Criança

\section{INTRODUÇÃO}

As habilidades em consciência fonológica (CF) vêm sendo alvo de muitos estudos ${ }^{1-3}$ e têm des-

(1) Fonoaudióloga; Policlínica Sarandi, Sarandi, RS; Especialista em Fonoaudiologia Hospitalar pelo Centro Universitário São Camilo; Mestre em Distúrbios da Comunicação Humana pela Universidade Federal de Santa Maria.

(2) Fonoaudióloga; Professora Associada do Curso de Fonoaudiologia e do Programa de Pós-Graduação em Distúrbios da Comunicação Humana da Universidade Federal de Santa Maria, UFSM, Santa Maria, RS; Doutora em Linguística Aplicada pela Pontifícia Universidade Católica do Rio Grande do Sul.

(3) Fonoaudióloga; Professora Adjunta do Curso de Fonoaudiologia e do Programa de Pós-Graduação em Distúrbios da Comunicação Humana da Universidade Federal de Santa Maria, UFSM, Santa Maria, RS; Doutora em Linguística Aplicada pela Pontifícia Universidade Católica do Rio Grande do Sul.

Conflito de interesses: inexistente pertado o interesse de pesquisadores de diferentes áreas para os aspectos relacionados ao seu desenvolvimento e influências na aquisição da linguagem oral e escrita.

Há na literatura várias pesquisas envolvendo a variável sexo, relacionada às diferenças anatômicas e funcionais do cérebro ${ }^{4}$; desvios fonológicos ${ }^{5}$; desenvolvimento da linguagem ${ }^{6}$; aprendizagem ${ }^{4}$, e dificuldades de aprendizagem ${ }^{7-9}$, evidenciando diferenças importantes. Porém, são escassas as pesquisas que consideram a variável sexo e a CF como foco principal da investigação.

Em estudos recentes ${ }^{3,10}$, foi verificado que meninas possuem maior habilidade para analisar pequenos segmentos, já os meninos desempenharam melhor as tarefas de análise com palavras.

Pesquisar como o desenvolvimento das habilidades em CF acontece em cada sexo possibilitaria maior compreensão sobre o processo e uma abordagem com orientações específicas a cada caso ${ }^{10}$. 
A CF não surge de uma hora para outra ${ }^{11}$, mas desenvolve-se gradualmente durante a infância ${ }^{12,13}$ como parte da consciência linguística ${ }^{13}$. Parece que essa habilidade poderia ser favorecida pelo bilinguismo ${ }^{14-18}$, tema que também tem merecido destaque nos últimos anos ${ }^{14,19-25}$.

Acredita-se que um ambiente bilíngue de aquisição da linguagem favoreça o desenvolvimento de habilidades perceptuais que habilitarão a criança para a distinção entre as suas duas línguas. Além disso, um ambiente de input linguístico rico teria como consequência a habilidade em discriminar fonemas ${ }^{26}$.

Ainda, estudos ${ }^{23,27}$ que investigaram a relação entre o bilinguismo e habilidades metalinguísticas, dentre elas a consciência fonológica (CF), mostraram haver correlações positivas no desempenho de bilíngues nessas tarefas

Diante das possíveis relações entre CF, bilinguismo e sexo, este estudo pretendeu investigar o desempenho de meninas e meninos, mono e bilíngues, em habilidades de CF.

\section{MÉTODOS}

Para desenvolver a pesquisa, foram contatadas 21 escolas do município de Agudo, interior do Rio Grande do Sul (RS) (16 municipais, três estaduais e duas particulares), sendo expostos os objetivos bem como a importância do trabalho no contexto educacional.

Para esse estudo, um questionário foi elaborado pelas pesquisadoras com a finalidade de coletar informações sobre os alunos e incluí-los nos requisitos necessários para a participação na pesquisa. Baseado em anamneses de rotina na área da linguagem, o questionário abordou perguntas objetivas e de fácil entendimento sobre o desenvolvimento geral da criança (pré, peri e pós-natal) até o período escolar; constelação familiar e idioma(s) que costumam usar e questões acerca da(s) língua(s) a que foi exposta, desde que idade foi exposta, bem como o(s) idioma(s) que a criança domina.

Foram enviados 1200 questionários e Termos de Consentimento Livre e Esclarecido - TCLE - aos responsáveis legais dos alunos de quatro a oito anos de idade, distribuídos entre as 21 escolas do município e retornaram apenas 632 de 16 escolas (11 municipais, três estaduais e duas particulares).

Para selecionar a amostra, foi realizada a análise dos questionários devolvidos considerandose os seguintes critérios de inclusão: crianças que estavam autorizadas pelos responsáveis a participarem da pesquisa; sem história significativa de deficiência auditiva, visual, neurológica e emocional; sem história de doença congênita, de atraso no desenvolvimento geral e queixas de dificuldades ou transtornos de aprendizagem, linguagem expressiva e compreensiva; possuir idade entre quatro e oito anos; ser monolíngue (português) ou bilíngue (alemão e português).

As crianças pré-selecionadas a partir dos questionários passaram por triagem auditiva baseada em resolução do Conselho Federal de Fonoaudiologia ${ }^{28}$. Foram submetidas à inspeção do meato acústico externo de cada orelha, por meio de um otoscópio, para verificar a presença, ou não, de corpos estranhos, de tampão ou excesso de cerúmen. Aquelas que apresentaram algum tipo de alteração foram encaminhadas ao médico otorrinolaringologista e excluídas da pesquisa. Foi realizada triagem auditiva por via aérea com um Audiômetro (marca Danplex, modelo DA 65), devidamente calibrado, na própria escola, em sala silenciosa, quando foram testadas as frequências de 1000 a $4000 \mathrm{~Hz}$, em $20 \mathrm{~dB}$ NA. As crianças que apresentaram desempenho negativo na triagem auditiva após duas testagens na mesma sessão e retestagem em nova oportunidade foram eliminadas do estudo e devidamente encaminhadas a atendimento específico.

Assim, foram excluídas as crianças que não obtiveram autorização para participar da pesquisa; apresentaram problemas pré, peri e pós-natais que pudessem ter provocado condições incompatíveis com o adequado desenvolvimento neuropsicomotor, de visão, de fala e da audição e que falharam na triagem auditiva.

Por fim, a amostra totalizou 89 sujeitos, com faixa etária entre quatro anos e um mês e oito anos e onze meses (sendo 42 do sexo masculino e 47 do sexo feminino). Ainda, dividiu-se a amostra em Grupo Monolíngue (GM) - com sujeitos expostos regularmente à fala do português desde o nascimento até os estágios finais da coleta de dados do estudo, e faixa etária entre quatro anos e um mês e oito anos e 11 meses, sendo 19 do sexo masculino e 23 do sexo feminino; e Grupo Bilíngue (GB) - com sujeitos expostos regularmente à fala do alemão $\mathrm{e}$ do português desde o nascimento até os estágios finais da coleta de dados do estudo e faixa etária entre quatro anos e quatro meses e oito anos e nove meses (sendo 23 do sexo masculino e 24 do sexo feminino).

Salienta-se que os sujeitos bilíngues selecionados foram expostos regularmente à conversação dos dois sistemas linguísticos (alemão e português) no contexto familiar e que a rede de ensino de Agudo/RS não pratica a educação bilíngue.

À amostra foi aplicado o Protocolo de Tarefas de CF ${ }^{12}$ padronizado por Cielo (2001) ${ }^{12}$ e já utilizado em outras populações ${ }^{3,10}$. A fim de verificar o desempenho em habilidades de CF, a autora ${ }^{12}$ 
propõe a realização de 12 tipos de atividades: segmentação de frases em palavras; realismo nominal; detecção de rimas; síntese silábica; segmentação silábica; detecção de sílabas; reversão silábica; exclusão fonêmica; detecção de fonemas; síntese fonêmica; segmentação fonêmica e reversão fonêmica. Levando-se em consideração a idade do aluno, o protocolo prevê diferentes graus de complexidade quanto à natureza das tarefas, e também diferentes graus de complexidade dentro de cada uma como o número e tipo de palavras, número de sílabas e fonemas.

Como cada item dentro de cada tarefa representa certo grau de complexidade, os pontos foram computados conforme as subdivisões de cada tarefa, com um máximo de dez pontos para cada subtipo.

Em sala silenciosa disponibilizada pela escola, o protocolo foi aplicado individualmente e utilizada linguagem simples. Para descartar a hipótese de a criança poder se sentir testada - o que poderia gerar certo nível de ansiedade frente às atividades, afirmou-se que se tratava de um "jogo", e não de um "teste" e que não seria necessário acertar tudo. Um único avaliador investigou o desempenho de toda a amostra do estudo.

Cada tarefa foi precedida por um treino e cada item da tarefa pôde ser realizado com duas tentativas. O treino teve como finalidade a compreensão da tarefa, evitando que questões de semântica ou memória interferissem na realização da tarefa propriamente dita. Caso o acerto ocorresse na primeira tentativa de resposta, seriam computados dois pontos; se ocorresse na segunda, um ponto; e zero, se a criança errasse em ambas as tentativas. A tarefa era suspensa quando ocorressem três falhas sucessivas. As respostas de cada sujeito foram transcritas para um gabarito.

Este trabalho foi desenvolvido após a aprovação pelo Comitê de Ética em Pesquisa (CEP) da instituição de origem (0050.0.243.000-06), autorização institucional das escolas e autorização dos responsáveis pelos sujeitos da pesquisa mediante assinatura do termo de consentimento livre e esclarecido (CONEP 196/96).

Foram considerados todos os protocolos aplicados e a pontuação de toda a amostra foi tabulada em um banco de dados. Para uma análise quantitativa dos dados, realizou-se o teste estatístico de Kruskal-Wallis com $p=0,05$ quando se comparou $o$ desempenho de meninos e meninas monolíngues, bem como o desempenho de meninos e meninas bilíngues por tarefa de CF. Os resultados significantes foram assinalados com um asterisco.

\section{RESULTADOS}

$\mathrm{Na}$ Tabela 1, apresentam-se os resultados obtidos pelos sujeitos bilíngues do sexo masculino e do sexo feminino. Nessa análise, percebe-se a superioridade das meninas bilíngues em relação aos meninos bilíngues, na maioria das habilidades em $\mathrm{CF}$, com resultados significativos em duas tarefas: detecção de rima com trissílabos e síntese com quatro fonemas.

A Tabela 2 apresenta a análise dos sujeitos monolíngues, comparando o desempenho de meninos e meninas em habilidades de CF e apontando resultados estatisticamente equilibrados.

\section{DISCUSSÃO}

Comparando as achados obtidos na presente pesquisa observou-se que houve $100 \%$ de êxito por meninas e meninos no que se refere à tarefa de síntese silábica com dissílabos e trissílabos (T4), concordando com os resultados encontrados em dois estudos ${ }^{29,30}$ que utilizaram o mesmo instrumento de avaliação da CF que a presente pesquisa e corroborando os resultados de outra pesquisa ${ }^{31}$ que utilizou outro instrumento de avaliação da CF.

Correlacionando os resultados das habilidades em CF com a variável sexo, um dos estudos citados acima ${ }^{30}$ mostrou que ambos os sexos obtiveram $100 \%$ dos acertos nas provas de síntese e segmentação silábica. Em outro estudo ${ }^{29}$, todos os sujeitos obtiveram êxito na tarefa de síntese silábica com duas sílabas. Duas pesquisas ${ }^{30,32}$ observaram que ambos os sexos obtiveram $100 \%$ dos acertos nas provas de síntese e segmentação silábica. Em outra ${ }^{12}$, essas tarefas também apresentaram uma média elevada de acertos. Verificando as tabelas deste estudo, nota-se que meninos e meninas obtiveram escores altos, com $100 \%$ de acertos nas tarefas síntese silábica com dissílabos e trissílabos (T4_dissílabos e trissílabos) e nas tarefas de síntese silábica com quadrissílabos (T4_quadrissílabos) e segmentação silábica (T5) aproximaram-se da totalidade de acertos.

A consciência silábica, que envolve habilidades como síntese e segmentação silábica, é uma manipulação consciente e já aparece antes da alfabetização e das habilidades em consciência fonêmica 33 .

Os resultados (Tabela 1) apontam a tendência feminina de lidar melhor com a consciência fonêmica, ou seja, com as unidades menores, corroborando os resultados de estudos recentes ${ }^{3,10}$, que também verificaram tal tendência nas meninas.

A exclusão de fonemas (T8) e a reversão fonêmica (T12) são tarefas complexas que exigem alto 
Tabela 1 - Resultados obtidos entre as meninas e os meninos bilíngues no desempenho das habilidades em consciência fonológica

\begin{tabular}{|c|c|c|c|c|c|c|}
\hline \multicolumn{2}{|c|}{ TAREFA DE CF } & \multicolumn{2}{|c|}{$\begin{array}{l}\text { MENINAS } \\
\text { BILÍNGUES }\end{array}$} & \multicolumn{2}{|c|}{$\begin{array}{l}\text { MENINOS } \\
\text { BILÍNGUES }\end{array}$} & \multirow{2}{*}{$\mathbf{p}$} \\
\hline IAREFA D & $=\mathrm{Cr}$ & média & $\begin{array}{l}\text { Desvio- } \\
\text { padrão }\end{array}$ & média & $\begin{array}{l}\text { Desvio- } \\
\text { padrão }\end{array}$ & \\
\hline \multirow{6}{*}{$\begin{array}{l}\text { Segmentação de } \\
\text { frases em } \\
\text { palavras } \\
\text { (T1) }\end{array}$} & T1_2 palavras & 8,7083 & 1,9444 & 8,6086 & 2,4446 & 0,8855 \\
\hline & T1_3 palavras & 9,0500 & 1,6050 & 8,1333 & 3,3352 & 0,9539 \\
\hline & T1_4 palavras & 8,4000 & 1,9303 & 8,2000 & 3,1892 & 0,5896 \\
\hline & T1_5 palavras & 8,5294 & 2,2393 & 8,3333 & 3,0251 & 0,8386 \\
\hline & T1_6 palavras & 8,8823 & 1,5363 & 8,0833 & 2,9682 & 0,5388 \\
\hline & T1_7 palavras & 8,2941 & 2,1437 & 7,5833 & 3,0289 & 0,4686 \\
\hline Realismo nominal (T2) & T2 & 9,3333 & 1,2394 & 8,9565 & 1,223 & 0,1382 \\
\hline \multirow{2}{*}{$\begin{array}{c}\text { Detecção de rimas } \\
\text { (T3) }\end{array}$} & T3_dissílabos & 8,4583 & 1,7189 & 8,2727 & 1,6954 & 0,6411 \\
\hline & T3_trissílabos & 8,8750 & 1,6550 & 7,6363 & 1,6774 & $0,0087^{*}$ \\
\hline \multirow{3}{*}{ Síntese silábica (T4) } & T4_dissílabos & 10 & 0 & 10 & 0 & 1,0000 \\
\hline & T4_trissílabos & 10 & 0 & 10 & 0 & 1,0000 \\
\hline & T4_quadrissílabos & 10 & 0 & 9,9565 & 0,2085 & 0,3070 \\
\hline \multirow{3}{*}{ Segmentação silábica (T5) } & T5_dissílabos & 9,8333 & 0,4815 & 9,8695 & 0,4576 & 0,6908 \\
\hline & T5_trissílabos & 9,5416 & 0,8329 & 9,6086 & 0,7827 & 0,7840 \\
\hline & T5_quadrissílabos & 9,1250 & 1,6500 & 9,3478 & 1,1122 & 0,9370 \\
\hline \multirow{3}{*}{ Detecção de sílabas (T6) } & T6_inicial & 9,1666 & 1,4345 & 8,5909 & 1,8938 & 0,1634 \\
\hline & T6_final & 8,4166 & 1,7916 & 7,9545 & 2,2141 & 0,4476 \\
\hline & T6_medial & 8,3333 & 1,8097 & 7,7727 & 2,6173 & 0,5880 \\
\hline \multirow{3}{*}{ Reversão silábica (T7) } & T7_dissílabos & 8,1428 & 2,1974 & 8,1578 & 2,4779 & 0,7105 \\
\hline & T7_trissílabos & 7,5294 & 2,7412 & 7,8333 & 2,0816 & 0,9278 \\
\hline & T7_quadrissílabos & 6,1764 & 2,5306 & 4,9166 & 1,7298 & 0,1207 \\
\hline \multirow{3}{*}{ Exclusão de fonemas (T8) } & T8_inicial & 9,1764 & 1,8109 & 9 & 1,7580 & 0,7867 \\
\hline & T8_final & 9,4705 & 0,8744 & 9,0833 & 1,2401 & 0,3681 \\
\hline & T8_medial & 9,3529 & 1,5387 & 8,7500 & 1,5447 & 0,0670 \\
\hline \multirow{3}{*}{ Detecção de fonemas (T9) } & T9_inicial & 8,8333 & 1,7610 & 8,0909 & 2,1801 & 0,2245 \\
\hline & T9_final & 8,2380 & 1,8948 & 7,5263 & 2,4122 & 0,3262 \\
\hline & T9_medial & 8 & 2 & 6,7666 & 3,1217 & 0,2742 \\
\hline \multirow{4}{*}{$\begin{array}{c}\text { Síntese } \\
\text { fonêmica (T10) }\end{array}$} & T10_3 fonemas & 9,5882 & 0,6183 & 9,0833 & 1,3113 & 0,3989 \\
\hline & T10_4 fonemas & 9,5882 & 0,8702 & 8,5000 & 1,6787 & $0,0219^{*}$ \\
\hline & T10_5 fonemas & 7,7058 & 2,0237 & 6,4166 & 1,6213 & 0,0767 \\
\hline & T10_6 fonemas & 7,1764 & 2,1574 & 6,1666 & 1,1146 & 0,2237 \\
\hline \multirow{5}{*}{$\begin{array}{l}\text { Segmentação } \\
\text { fonêmica } \\
\text { (T11) }\end{array}$} & T11_3 fonemas & 9,0588 & 1,5996 & 9,3333 & 0,9847 & 0,8340 \\
\hline & T11_4 fonemas & 9,4117 & 1,2277 & 9,7500 & 0,8660 & 0,3129 \\
\hline & T11_5 fonemas & 8,5294 & 2,2112 & 9,5833 & 1,1645 & 0,0891 \\
\hline & T11_6 fonemas & 9 & 1,7056 & 8,6666 & 1,1180 & 0,2558 \\
\hline & T11_7 fonemas & 9 & 1,4770 & 8,8888 & 1,8333 & 0,7821 \\
\hline Reversão fonêmica (T12) & T12_2 e 3 fonemas & 7,6470 & 2,3168 & 6,4166 & 2,3532 & 0,1379 \\
\hline
\end{tabular}

* Valor estatisticamente significante (Kruskal-Wallis com $p=0,05$ ) 
Tabela 2 - Resultados obtidos entre as meninas e os meninos monolíngues no desempenho das habilidades em consciência fonológica

\begin{tabular}{|c|c|c|c|c|c|c|}
\hline \multicolumn{2}{|c|}{ TAREFA DE CF } & \multicolumn{2}{|c|}{$\begin{array}{c}\text { MENINAS } \\
\text { MONOLÍNGUES }\end{array}$} & \multicolumn{2}{|c|}{$\begin{array}{c}\text { MENINOS } \\
\text { MONOLÍNGUES }\end{array}$} & \multirow{2}{*}{$\mathbf{p}$} \\
\hline & LI & média & $\begin{array}{l}\text { Desvio- } \\
\text { padrão }\end{array}$ & média & $\begin{array}{l}\text { Desvio- } \\
\text { padrão }\end{array}$ & \\
\hline \multirow{6}{*}{$\begin{array}{l}\text { Segmentação de } \\
\text { frases em } \\
\text { palavras } \\
\text { (T1) }\end{array}$} & T1_2 palavras & 8,3913 & 1,8766 & 8,0526 & 2,3445 & 0,8334 \\
\hline & T1_3 palavras & 8,0952 & 2,1190 & 7,8823 & 1,7636 & 0,5370 \\
\hline & T1_4 palavras & 7,9047 & 2,1425 & 7,5294 & 1,8411 & 0,4376 \\
\hline & T1_5 palavras & 7,8333 & 2,7706 & 7,5882 & 2,6706 & 0,6597 \\
\hline & T1_6 palavras & 6,8333 & 2,4793 & 7,1176 & 2,8697 & 0,6277 \\
\hline & T1_7 palavras & 6 & 3,5809 & 6,7647 & 3,3824 & 0,5700 \\
\hline Realismo nominal (T2) & T2 & 9 & 1,2060 & 9,2105 & 1,2727 & 0,4572 \\
\hline \multirow{2}{*}{$\begin{array}{c}\text { Detecção de rimas } \\
\text { (T3) }\end{array}$} & T3_dissílabos & 7,3913 & 2,2307 & 7,7222 & 2,0235 & 0,7987 \\
\hline & T3_trissílabos & 7,8695 & 2,0069 & 7,4444 & 1,8541 & 0,4156 \\
\hline \multirow{3}{*}{ Síntese silábica (T4) } & T4_dissílabos & 10 & 0 & 10 & 0 & 1,0000 \\
\hline & T4_trissílabos & 10 & 0 & 10 & 0 & 1,0000 \\
\hline & T4_quadrissílabos & 9,9130 & 0,2881 & 10 & 0 & 0,1931 \\
\hline \multirow{3}{*}{ Segmentação silábica (T5) } & T5_dissílabos & 9,9565 & 0,2085 & 9,7894 & 0,5353 & 0,2056 \\
\hline & T5_trissílabos & 9,5652 & 1,2367 & 9,6842 & 0,7492 & 0,9008 \\
\hline & T5_quadrissílabos & 9 & 1,8340 & 9,5263 & 1,2187 & 0,3023 \\
\hline \multirow{3}{*}{ Detecção de sílabas (T6) } & T6_inicial & 7,9565 & 2,0333 & 8,4444 & 1,9769 & 0,3500 \\
\hline & T6_final & 7,0434 & 2,2858 & 7,7222 & 2,2701 & 0,2874 \\
\hline & T6_medial & 6,6956 & 2,3048 & 6,7222 & 2,5622 & 0,8008 \\
\hline \multirow{3}{*}{ Reversão silábica (T7) } & T7_dissílabos & 7,1000 & 3,5377 & 7,3333 & 2,9305 & 0,8464 \\
\hline & T7_trissílabos & 5,4444 & 3,5016 & 5,4117 & 3,6065 & 0,9735 \\
\hline & T7_quadrissílabos & 3,9444 & 2,9400 & 3,9411 & 2,7719 & 0,9200 \\
\hline \multirow{3}{*}{ Exclusão de fonemas (T8) } & T8_inicial & 7,1111 & 4,0276 & 6,8823 & 3,4978 & 0,6293 \\
\hline & T8_final & 7,7222 & 3,0254 & 8,0588 & 3,2686 & 0,6954 \\
\hline & T8_medial & 7,5555 & 3,9440 & 7,7647 & 2,7957 & 0,3344 \\
\hline \multirow{3}{*}{ Detecção de fonemas (T9) } & T9_inicial & 7,3913 & 2,4259 & 8,0555 & 2,4845 & 0,3336 \\
\hline & T9_final & 7,0500 & 2,2589 & 6,4444 & 2,9149 & 0,6773 \\
\hline & T9_medial & 5,7777 & 3,3352 & 6,1176 & 2,8697 & 0,8810 \\
\hline \multirow{4}{*}{$\begin{array}{c}\text { Síntese } \\
\text { fonêmica (T10) }\end{array}$} & T10_3 fonemas & 8,0555 & 2,3129 & 7,5882 & 2,7399 & 0,5186 \\
\hline & T10_4 fonemas & 7,7777 & 2,5565 & 7,9411 & 2,3040 & 0,7229 \\
\hline & T10_5 fonemas & 5,3333 & 2,8284 & 5,1176 & 2,6192 & 0,6850 \\
\hline & T10_6 fonemas & 4,5000 & 3,3121 & 4,5882 & 2,9803 & 0,8277 \\
\hline \multirow{5}{*}{$\begin{array}{l}\text { Segmentação } \\
\text { fonêmica } \\
\text { (T11) }\end{array}$} & T11_3 fonemas & 7,3888 & 3,8061 & 7,4705 & 2,8748 & 0,4043 \\
\hline & T11_4 fonemas & 7,6666 & 3,8501 & 7,1764 & 2,8990 & 0,1135 \\
\hline & T11_5 fonemas & 6,8333 & 4,2043 & 6,0588 & 3,2686 & 0,1643 \\
\hline & T11_6 fonemas & 6,8461 & 3,5319 & 6 & 3,1269 & 0,3974 \\
\hline & T11_7 fonemas & 6,9230 & 4,1324 & 5,6000 & 2,4585 & 0,1159 \\
\hline Reversão fonêmica (T12) & T12_2 e 3 fonemas & 5 & 3,6299 & 4,8235 & 3,0462 & 0,8548 \\
\hline
\end{tabular}

Kruskal-Wallis com $p=0,05$ 
nível de consciência fonêmica ${ }^{19}$. Nota-se que as meninas bilíngues (Tabela 4.1) foram soberanas no desempenho de todas as tarefas de exclusão de fonemas (T8), sendo que o bilinguismo parece ter sido o fator que reforçou os achados da variável sexo feminino.

Entre os sujeitos monolíngues, a análise dos resultados não apontou significância estatística no confronto entre meninas e meninos em nenhuma das tarefas de CF e os resultados encontram-se mais equilibrados, porém com tendência à superioridade feminina, confirmando os achados da literatura ${ }^{3,10}$.

Outros estudos ${ }^{34,35}$ que utilizaram um instrumento de avaliação da CF diferente da presente pesquisa também mostraram não haver diferenças significativas no desempenho de meninas e meninas nas habilidades em CF. Porém, a análise das duas tabelas corrobora os achados de uma recente pesquisa ${ }^{3}$ quando indica haver uma superioridade feminina nas tarefas de consciência fonêmica.

As tarefas de consciência fonêmica exigem análise e têm um grau de complexidade mais elevado, e o desempenho superior das meninas se poderia tentar justificar com o argumento de que as habilidades em consciência fonêmica são diretamente dependentes do domínio do código alfabético ${ }^{12} \mathrm{e}$, parece que o sexo feminino apresenta desenvolvimento mais rápido do código alfabético ${ }^{10}$, que é melhor a habilidade feminina no aprendizado da linguagem escrita ou porque a linguagem é processada de formas distintas no cérebro de masculino e feminino ${ }^{36}$. Parece que o sexo masculino faria uso de um hemisfério de cada vez, enquanto que o feminino usaria ambos simultaneamente ${ }^{4}$.

Pesquisas envolvendo CF e a variável sexo são escassas e, em sua grande maioria, não apresentam comparações do desempenho dos sujeitos com a utilização de testes estatísticos.

As informações encontradas na literatura recente ${ }^{3,10}$, que também realizou o tratamento estatístico dos dados, reforçaram a tendência apontada pelo presente estudo: a superioridade do sexo feminino no desempenho das habilidades em tarefas de CF.

Salienta-se que o bilinguismo foi um fator que manteve os resultados destacados anteriormente e que favoreceu a presença de resultados estatisticamente significantes (Tabela 1) para as tarefas de detecção de rima com trissílabos e síntese com quatro fonemas, não encontrados quando analisados somente sujeitos monolíngues.

\section{CONCLUSÃO}

Este estudo permitiu concluir que as meninas bilíngues foram superiores aos meninos bilíngues na maioria das tarefas em CF. Quando analisados os sujeitos monolíngues, houve equilíbrio estatístico nos resultados das habilidades em CF.

De maneira geral, há tendência à superioridade feminina no desempenho de tarefas em CF.

$\mathrm{O}$ estudo buscou colaborar com a literatura nacional ao incluir a variável bilinguismo como tema de investigação e pretendeu instigar a necessidade da continuidade de pesquisas futuras com amostras maiores e diferentes sistemas linguísticos.

\section{ABSTRACT}

Purpose: to investigate monolingual and bilingual girls and boys performance, in the skills of phonological awareness. Methods: questionnaire and hearing screening to select the sample, consisting of 89 students, aged 4:1 to 8:11-years old, 47 girls and 42 boys, of which 47 subjects were bilingual (Portuguese and German) and 42 monolingual (Portuguese). The selected sample was submitted to the assessment of skills in phonological awareness, applying the Phonological Awareness Protocol (Cielo, 2001) that suggests segmentation of sentences in words, nominal realism, rhyme detection, syllabic synthesis and segmentation, syllable detection, syllabic and phonemic reversion, phonemic exclusion, phoneme detection and phonemic synthesis and segmentation. For a quantitative data analysis, a statistical test of Kruskal-Wallis with $p=0.05$ was taken when comparing the monolingual boys' and girls' performance, and the bilingual boys' and girls' performance by task of phonological awareness. Results: when the bilingual subjects were analyzed, there was a statistical significance in the detection of rhyme with trisyllable $(p=0,0087)$ and synthesis of four phonemes $(p=0,0219)$, with advantage of girls, while in the analysis that compared monolingual boys and girls, the results were more balanced. Conclusions: despite of almost lacking statistically significant results in the comparison among bilingual and monolingual boys and girls, female superiority was observed in most of phonological awareness tasks.

KEYWORDS: Language; Language Arts; Multilingualism; Awareness; Child 


\section{REFERÊNCIAS}

1. Carroll JM, Snowling MJ, Hulme C, Stevenson

J. The development of phonological awareness in preschool children. Develop Psychol. 2003; 39(5):913-23.

2. Mota HB, Melo Filha MGC, Lasch SS. A consciência fonológica e o desempenho na escrita sob ditado de crianças com desvio fonológico após realização de terapia fonoaudiológica. Rev CEFAC. 2007; 9(4):477-82. http://dx.doi.org/ S1516-18462007000400007

3. Moura SRS. Os efeitos do programa de estimulação e a variável sexo no desempenho em consciência fonêmica [dissertação]. Santa Maria (RS): Universidade Federal de Santa Maria; 2008.

4. Preuschoff G. Criando meninas. São Paulo: Fundamento; 2003.

5. Farias SR, Ávila CRB, Vieira MM. Relação entre fala, tônus e praxia não-verbal do sistema estomatognático em pré-escolares. Pró-Fono. 2006; 18(3):267-76.

6. Mezzomo CL. Aquisição da coda no português brasileiro: uma análise via teoria de princípios e parâmetros. [tese]. Porto Alegre (RS): Pontifica Universidade Católica do Rio Grande do Sul Faculdade de Letras; 2003.

7. Bandeira M, Rocha SS, Souza TMP, Del Prette ZAP, Del Prette A. Comportamentos problemáticos em estudantes do ensino fundamental: características da ocorrência e relação com habilidades sociais e dificuldades de aprendizagem. Est Psicol. 2005; 11(2):199-208.

8. Vieira RMR, Grosso PMP, Zorzi JL, Chiappetta ALML. Estudos comparativos sobre a influência da leitura nos erros da escrita entre meninos e meninas de quarta série. Rev CEFAC. 2006; 8(3):281-8.

9. Capellini SA, Padula NAMR, Santos LCA, Lourenceti MD, Carrenho EH, Ribeiro LA. Desempenho em consciência fonológica, memória operacional, leitura e escrita na dislexia familial. Pró-Fono. 2007; 19(4):374-80.

10. Andreazza-Balestrin C. Relação entre desempenho em consciência fonológica e a variável sexo, na infância [dissertação]. Santa Maria (RS): Universidade Federal de Santa Maria; 2007.

11. Cielo CA. A avaliação das habilidades em consciência fonológica. J Bras Fonoaudiol. 2003; 4(16):163-74.

12. Cielo CA. Habilidades em consciência fonológica em crianças de 4 a 8 anos de idade [tese]. Porto Alegre (RS): Pontifica Universidade Católica do Rio Grande do Sul; 2001.

13. Capellini SA, Ciasca SM. Comparação do nível de leitura entre escolares sem e com queixa de dificuldade na leitura. Rev Soc Bras Fonoaudiol. 1999; 5(5):32-6.

14. Carlisle JF, Beeman M, Davis LH, Spharim G. Relationship of metalinguistic capabilities and reading achievement for children who are becoming bilingual. Appl Psycholinguist. 1999; 20(4):459-78.

15. Bialystok $E$. Metalinguistic aspects of bilingual processing. Ann Rev Appl Linguist. 2001; 21:169-81.

16. Goetz PJ. The effects of bilingualism on theory of mind development. Biling Lang Cognit. 2003; 6(1):1-15.

17. Charkova KD. Early foreign language education and metalinguistic development: a study of monolingual, bilingual and trilingual children on noun definition tasks. Ann Rev Lang Acquis. 2004; 3(1):51-88.

18. Bialystok E, Craik FI, Klein R, Viswanathan M. Bilingualism, aging, and cognitive control: evidence from the Simon task. Psychol Aging. 2004; 19(2):290-303.

19. Bialystok E. Acquisition of literacy in bilingual children: a framework for research. Lang Learn. 2007; 57(1):45-77.

20. Bialystok E, Majumder S, Martin MM. Developing phonological awareness: is there a bilingual advantage? Appl Psycholing. 2003; 24(1):27-44.

21. Gutiérrez-Clellen VF, Simon-Cereijido G, Wagner C. Bilingual children with language impairment: a comparison with monolinguals and second language learners. Appl Psycholing. 2008; 29(1):3-19.

22. Chen ES. Language convergence and bilingual acquisition: the case of conditional constructions. Ann Rev Lang Acquis. 2004; 3(1):89-137.

23. Chen X, Anderson RC, Li W, Hao M, Wu X, Shu $\mathrm{H}$. Phonological awareness of bilingual and monolingual Chinese children. J Educ Psychol. 2004; 96(1):142-51.

24. Eviatar Z, Ibrahim R. Bilingual is as bilingual does: metalinguistic abilities of Arabic-speaking children. Appl Psycholing. 2000; 21(4):451-71.

25. Schmitz K, Müller N. Strong and clitic pronouns in monolingual and bilingual acquisition of French and Italian. Biling Lang Cognit. 2008; 11(1):19-41.

26. Rocca PDA. O desempenho de falantes bilíngues: evidências advindas da investigação do VOT de oclusivas surdas do inglês e do português. Delta. 2003; 19(2):303-28.

27. Loizou M, Stuart M. Phonological awareness in monolingual and bilingual English and Greek fiveyear-olds. J Res Read. 2003; 26(1):3-18.

28. Conselho Federal de Fonoaudiologia. CFFa Resolução 274. Brasília/DF; 2001.

29. Paula GR, Mota HB, Keske-Soares M. A terapia em consciência fonológica no processo de alfabetização. Pró-Fono. 2005; 17(2):175-84. 
30. Paes CTS, Pessoa ACRG. Habilidades fonológicas em crianças não alfabetizadas e alfabetizadas. Rev. CEFAC. 2005; 7(2):149-57.

31. Cavalcante CA, Mendes MAM. A avaliação da consciência fonológica em crianças de primeira série alfabetizadas com metodologias diferentes. Rev. CEFAC. 2003; 5(3):205-8.

32. Meneses MS, Lozi GP, Souza LR, AssencioFerreira VJ. Consciência fonológica: diferenças entre meninos e meninas. Rev. CEFAC. 2004; 6(3):242-6.

33. Alegria J, Pignot E, Morais J. Phonetic analysis of speech and memory codes in beginning readers.
In: 5th International Congress of the International Society of Applied Psycholinguistics. 25-27 junho/ 1997, Porto-Portugal, 1999.

34. Maluf MR, Barrera SD. Consciência fonológica e linguagem escrita em pré-escolares. Psicol Reflex Crít. 1997; 10(1):125-45.

35. Gindri G, Keske-Soares M, Mota HB. Memória de trabalho, consciência fonológica e hipótese de escrita. Pró-Fono. 2007; 19(3):313-22.

36. Salles JF, Mota HB, Cechella C, Parente MAMP. Desenvolvimento da consciência fonológica de crianças de primeira e segunda séries. Pró-Fono. 1999; 11(2):68-76.

RECEBIDO EM: 24/07/2008

ACEITO EM: 27/04/2009

Endereço para correspondência:

Sabrina Schützenhofer Lasch

Rua Germano Hentschke, 676

Agudo - RS

CEP: $96540-000$

E-mail: sabrinafono@bol.com.br 